\title{
Vital-Sign Synchrony as a Marker for Patient Circadian Rhythms in an Intensive Care Unit
}

\author{
Shaun Davidson ${ }^{1}$, Mauricio Villarroel ${ }^{1}$, Eoin Finnegan ${ }^{1}$, Mirae Harford ${ }^{1}$, Joao Jorge ${ }^{1}$, Lionel \\ Tarassenko $^{1}$ \\ ${ }^{1}$ University of Oxford, Oxford, United Kingdom
}

\begin{abstract}
Patient circadian rhythms are often disrupted in an intensive care unit (ICU). This disruption is associated with worsened patient outcomes, thus new methods are needed to quantify patient circadian rhythms. We hypothesise that the cross-correlation between vital-sign circadian profiles will allow us to stratify patients by rhythm strength, without reliance on a prior assumed rhythm profile.

We selected from the eICU-CRD and MIMIC-III databases the cohort of patients in their final 24 hours of ICU stay who subsequently recovered. We then calculated the mean cross-correlation $(R)$ between each patient's systolic blood pressure, heart rate, and respiratory rate profiles, and grouped them into 'high', 'mid', and 'low' correlation cohorts.

The high-corr. cohorts showed vital-sign profiles that closely resembled those reported in the literature for non-ICU cohorts, with peaks at awakening and in the evening, and a large trough overnight. The mid- and low-corr. cohorts, in contrast, showed less consistent and defined peaks and troughs. Vital-sign peak-nadir excursions in the high-correlation cohorts were greater, and the length of ICU stay significantly shorter $(p<0.05)$, than those for the low-corr. cohorts.
\end{abstract}

\section{Introduction}

Patient circadian rhythms are often disrupted in an intensive care unit (ICU) due to medication, sedation, inflammation and the ambient ICU environment [1]. This disruption is associated with worsened patient outcomes such as delirium [2] and mortality [3]. Abnormal sleep states and the requirement of additional instrumentation, such as electroencephalography (EEG, brain activity), make monitoring circadian rhythms using sleep difficult in the ICU [4]. Thus, new methods leveraging readily available ICU information for monitoring patient circadian rhythmicity are needed.
Circadian rhythms are known to exist in a variety of vital signs including blood pressure, temperature, respiratory rate and heart rate [5-7]. In this paper, we focus on cardio-respiratory vital signs with well-documented circadian behaviour commonly monitored in the ICU, specifically systolic blood pressure (SBP), heart rate (HR), and respiratory rate (RR). These vital signs typically exhibit an approximately sinusoidal daily pattern, with elevated levels during the day and a nocturnal dip [5,7]. Previous studies have found that atypical patterns in SBP, specifically a 'non-dipping' or 'reverse-dipping' pattern (elevated nocturnal SBP) are associated with an increased risk of myocardial infarction or stroke [8,9]. Traditionally, 'dipping', 'non-dipping', or 'reverse-dipping' behaviour are established using fixed thresholds (e.g. a $10 \%$ nocturnal decrease in SBP for 'dipping' [8]) and rely on arbitrary definitions of 'night' and 'day', both of which vary between studies $[5,8,9]$.

We hypothesise that patients who exhibit a 'dipping' pattern in SBP are likely to exhibit similar dipping patterns in other cardio-respiratory vital signs such as HR and RR. This is intuitive, as 'non-dipping' or 'reverse dipping' behaviour is thought to be associated with autonomic nervous system (ANS) disruption [8], which would affect other vital signs. However, this behaviour has not previously been shown. This work presents algorithms for the quantitative assessment of patient circadian rhythmicity independent of any assumed prior vital-sign profile or 'night' and 'day' period. While we have used hourly measurements for each vital-sign, this quantitative assessment can potentially be performed with fewer sample points due to including three cardio-respiratory vital signs, as opposed to only SBP.

\section{Methods}

\subsection{Data Selection}

The following databases were used for this study:

- Medical Information Mart for Intensive Care III (MIMIC-III), which contains critical care information 
gathered between 2001 and 2012 from the Beth Israel Deaconess Medical Centre (BIDMC) in Boston, MA, USA $[10,11]$.

- The eICU Collaborative Research Database (eICU-CRD), which contains critical care information gathered between 2014 and 2015 from 208 hospitals across the continental USA [12].

We applied the selection criteria detailed in [6] to these databases. These criteria focused on selecting the final 24 hours prior to discharge from an ICU of patients who were ultimately discharged home from the hospital after their ICU stay, and who were not readmitted within 6 months. This cohort was deemed most likely to exhibit typical circadian behaviour. The measurements used were cuff SBP, as well as HR and RR recorded by bedside monitor. Measurements taken while a patient was subject to vital-sign altering medication (e.g. vasopressors) were excluded, as were physiologically improbable measurements.

\subsection{Data Analysis}

To build the mean circadian vital-sign profiles, all measurements for each patient in each one-hourly left-aligned period were averaged for each vital-sign [6]. We then assessed the mean pairwise Pearson's cross correlation (R) between SBP, HR, and RR for the final 24 hours of ICU stay. The mean pairwise cross-correlation was determined:

$$
R=\frac{R_{S B P-H R}+R_{H R-R R}+R_{R R-S B P}}{3}
$$

We grouped the $20 \%$ of patients with the greatest mean pairwise cross-correlation as a 'high correlation' cohort, and the $20 \%$ of patients with the lowest mean pairwise cross-correlation as a 'low correlation' cohort. The remaining $60 \%$ of patients were placed in a 'mid correlation' cohort.

We then used two sample Student's t-tests to assess differences in the underlying demographics such as age, gender, ICU length of stay (LOS) and the Oxford Acute Severity of Illness Score (OASIS [13]) between the cohorts. The hourly $95 \%$ confidence interval of the vital-sign mean was calculated to provide an indication of intra-cohort variability. The peak - nadir excursion [6] of each vital-sign profile was calculated as an indication of rhythm amplitude or strength.

Given the well documented dependency of SBP, HR, and RR on age and gender, the cohorts were broken down into gender and age subgroups (as in [6]) to assess the methodology's ability to stratify 'dippers', 'non-dippers', and 'reverse-dippers' within these patient demographics.

\section{Results}

The cut-offs for the low corr. and high corr. cohorts were $\mathrm{R}=-0.02$ and 0.38 for MIMIC-III and $\mathrm{R}=-0.01$ and 0.47 for eICU-CRD. From table 1, across both databases, the high corr. cohort had a shorter LOS than the low corr. cohort ( $p<0.05)$. In eICU-CRD, the high corr. cohort had a lower OASIS $(\mathrm{p}<0.05)$ and age $(\mathrm{p}<0.05)$ than the low corr. cohort.

Table 1. Demographics of the patient cohorts.

\begin{tabular}{c|rrrrr}
\hline Cohort & \multicolumn{5}{|c}{ Demographics } \\
\hline MIMIC-III: & No. Stays & $\%$ Men & Age & LOS & OASIS \\
High Corr. & 2,374 & 60.5 & 58.2 & ${ }^{*} 2.8$ & 27.6 \\
Mid Corr. & 7,123 & 59.7 & 59.1 & 3.0 & 27.6 \\
Low Corr. & 2,374 & 57.8 & 57.7 & ${ }^{*} 3.1$ & 27.8 \\
\hline Overall & 11,872 & 59.4 & 58.6 & 3.0 & 27.6 \\
\hline eICU-CRD: & No. Stays & $\%$ Men & Age & LOS & OASIS \\
High Corr. & 6,952 & 55.3 & ${ }^{*} 57.4$ & ${ }^{*} 2.6$ & ${ }^{*} 26.5$ \\
Mid Corr. & 20,855 & 55.0 & 59.0 & 2.7 & 27.1 \\
Low Corr. & 6,952 & 54.5 & ${ }^{*} 58.4$ & ${ }^{*} 2.7$ & ${ }^{*} 27.3$ \\
\hline Overall & 35,143 & 54.7 & 58.4 & 2.7 & 26.9 \\
\hline
\end{tabular}

* Differences between high and low corr. cohorts, $\mathrm{p}<0.05$.

From table 2, the high corr. cohort consistently exhibited the greatest peak-nadir excursions across all databases and vital-signs.

Table 2. Peak - nadir excursions for the patient cohorts.

\begin{tabular}{l|rrr}
\hline \multicolumn{1}{c|}{ Cohort } & \multicolumn{3}{|c}{ Peak-Nadir Excursion } \\
\hline MIMIC-III: & SBP (mmHg) & HR (bpm) & RR (breath/min) \\
High Corr. & 9.8 & 7.8 & 2.8 \\
Mid Corr. & 4.9 & 5.1 & 1.4 \\
Low Corr. & 3.3 & 3.4 & 0.7 \\
\hline eICU-CRD: & SBP (mmHg) & HR (bpm) & RR (breath/min) \\
High Corr. & 11.6 & 8.5 & 3.4 \\
Mid Corr. & 5.1 & 5.7 & 1.8 \\
Low Corr. & 3.8 & 4.6 & 0.9 \\
\hline
\end{tabular}

Fig. 1 shows the overall vital-sign profiles for the selected patient cohorts, grouped by gender. Consistent 'reverse-dipping' in SBP and 'non-dipping' behaviour in RR was present in the low corr. cohort for both databases. HR profiles showed a more limited variability between the low corr. and high corr. cohorts. Figs. 2 and 3 show the vital-sign profiles for men and women, respectively, grouped by age. The high corr. cohort showed 'dipping' behaviour in all vital-signs that more closely correspond to circadian vital-sign profiles reported in the literature for non-ICU cohorts. The low corr. cohorts showed 'non-dipping' or 'reverse-dipping' behaviour in SBP and RR.

\section{Discussion}

Consistent nocturnal 'dipping' behaviour, which resembles circadian vital-sign profiles reported in the literature for non-ICU cohorts of patients [5,7], was exhibited in 

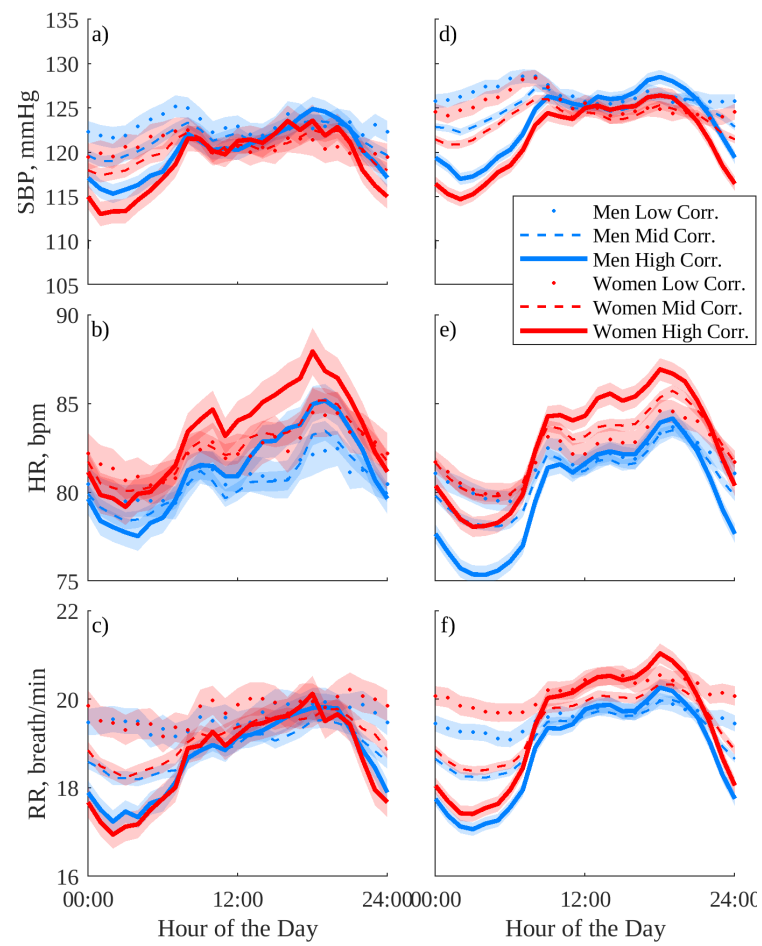

Figure 1. Vital-sign profiles categorised using the mean cross-correlation boundaries. MIMIC-III profiles for: a) SBP; b) HR; c) RR. eICU-CRD profiles for: d) SBP; e) HR; f) RR.

the high corr. cohorts across the various demographic subgroups in both MIMIC-III and eICU-CRD databases. In contrast, the low corr. cohorts demonstrated consistent 'non-dipping' or 'reverse-dipping' behaviour. This result supports the notion that the mean cross-correlation between cardio-respiratory vital-signs can be used to consistently isolate groups with typical or atypical circadian behaviour, across cohorts drawn from different locales, ages, or genders. Further evidence is provided in table 2 where the peak-nadir excursions in the high corr. cohort were consistently greater than those in the low corr. cohort in both databases (though the low corr. cohort included 'reverse-dippers', who may have large peak-nadir excursions). The peak-nadir excursions in the high corr. cohort also more closely resemble those reported in the literature for non-ICU cohorts [5, 7] (previous work has noted that circadian vital-sign profile amplitude is typically suppressed in an ICU [6]). Finally, the relative consistency in the $\mathrm{R}$ thresholds for the 'high', 'mid', and 'low' corr. cohorts suggests applicability across databases of threshold values in the vital-sign cross-correlation metric.

The statistically significant difference in patient LOS in both databases between the low and high corr. cohorts

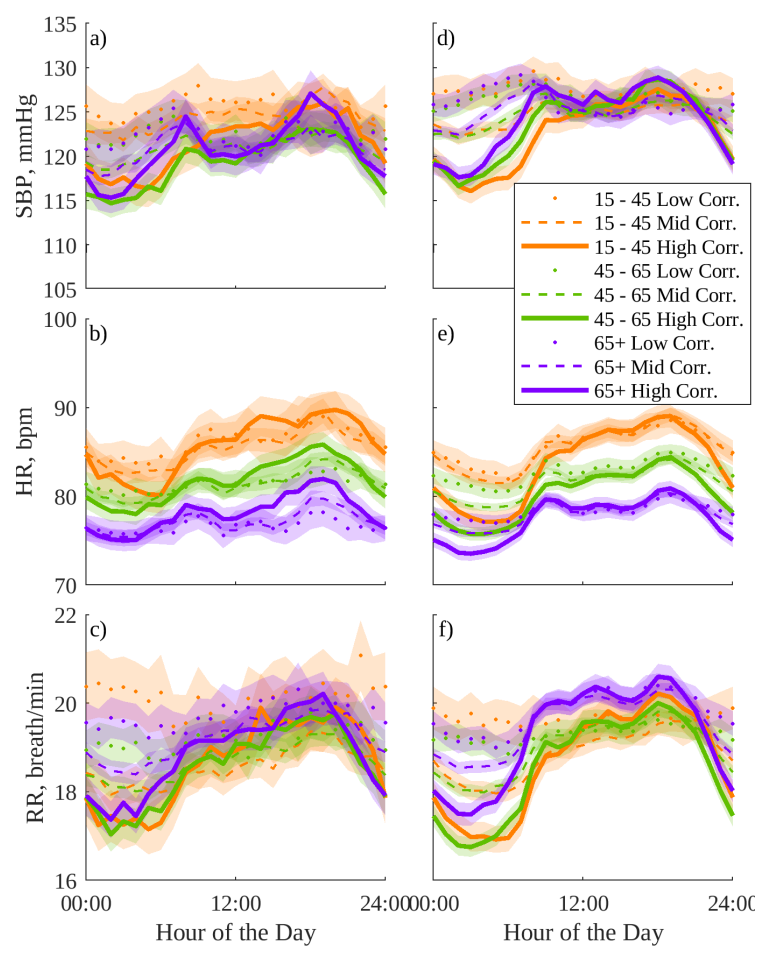

Figure 2. Vital-sign profiles in men categorised using the mean cross-correlation boundaries. MIMIC-III profiles for: a) SBP; b) HR; c) RR. eICU-CRD profiles for: d) SBP; e) HR; f) RR.

suggests that these cohorts exhibit differing clinical presentation. This result corresponds well with the notion that there is an association between disrupted patient circadian rhythms and an increased LOS in the ICU [1].

There are several implications of the presented results. First, they imply that the broader ANS effects associated with the 'non-dipping' or 'reverse-dipping' pattern manifest in disrupted HR and RR behaviour. Second, that the mean cross-correlation of cardio-respiratory vital-signs naturally stratifies patients into 'dipper', 'non-dipper', and 'reverse-dipper' categories suggests it has potential as a quantitative metric for assessing patient circadian rhythmicity. Such a metric would be independent of any assumed prior vital sign profile, which is a limitation of approaches such as cosinor analysis [5]. Introducing additional, readily available vital-signs to the process of classifying an individual's circadian rhythms also potentially allows for categorisation using fewer measurements across multiple vital signs.

There are some limitations to this study. Both databases are from the US, so there is limited diversity in demographics and clinical practice. Both databases were collected retrospectively during routine care, rather than as 

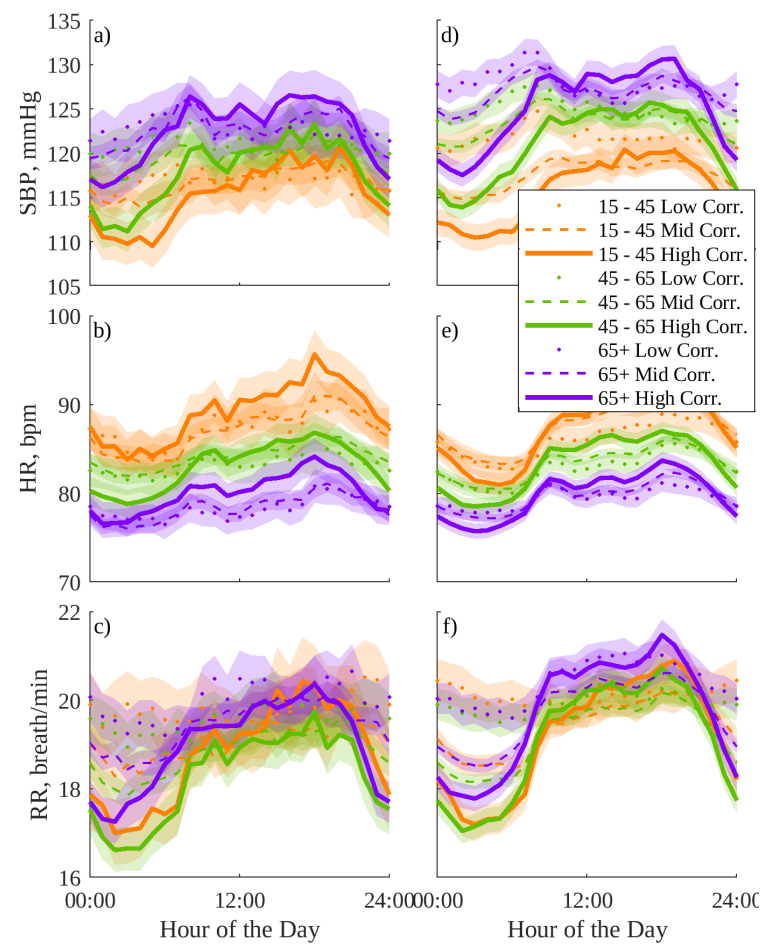

Figure 3. Vital-sign profiles in women categorised using the mean cross-correlation boundaries. MIMIC-III profiles for: a) SBP; b) HR; c) RR. eICU-CRD profiles for: d) SBP; e) HR; f) RR.

part of a study designed to analyse circadian rhythmicity. Finally, all of the results in this study are reported for cohorts or groups of patients, rather than for individuals.

\section{Conclusion}

We have shown that the cross-correlation between cardio- respiratory vital-signs can be used to stratify patients who show 'dipping' as opposed to 'non-dipping' or 'reverse-dipping' SBP behaviour. We have further shown that the 'dipping' patients have vital-sign amplitudes that more closely resemble healthy behaviour, and a statistically significant decrease in LOS. Overall, these results suggest that the cross-correlation between cardio-respiratory vital signs has potential as a quantitative metric to assess patient circadian rhythmicity in the ICU.

\section{References}

[1] Papaioannou V, Mebazaa A, Plaud B, Legrand M. 'chronomics' in icu: circadian aspects of immune response and therapeutic perspectives in the critically ill. Intensive care medicine experimental 2014;2(1):18.

[2] Estrup S, Kjer C, Poulsen L, Gogenur I, Mathiesen O. Delirium and effect of circadian light in the intensive care unit: a retrospective cohort study. Acta Anaesthesiologica Scandinavica 2018;62(3):367-375.

[3] Li J, Li R, Gao Y, Zhang J, Zhao Y, Zhang X, Wang G. Nocturnal mean arterial pressure rising is associated with mortality in the intensive care unit: A retrospective cohort study. Journal of the American Heart Association 2019; 8(19):e012388.

[4] Watson PL, Pandharipande P, Gehlbach BK, Thompson JL, Shintani AK, Dittus BS, Bernard GR, Malow BA, Ely EW. Atypical sleep in ventilated patients: empirical electroencephalography findings and the path toward revised icu sleep scoring criteria. Critical care medicine 2013;41(8).

[5] Hermida RC, Ayala DE, Fernández JR, Mojón A, Alonso I, Calvo C. Modeling the circadian variability of ambulatorily monitored blood pressure by multiple-component analysis. Chronobiology international 2002;19(2):461-481.

[6] Davidson S, Villarroel M, Harford M, Finnegan E, Jorge J, Young D, Watkinson P, Tarassenko L. Vital-sign circadian rhythms in patients prior to discharge from an icu: a retrospective observational analysis of routinely recorded physiological data. Critical Care 2020;24(1):1-13.

[7] Bosco G, Ionadi A, Panico S, Faralli F, Gagliardi R, Data P, Mortola JP. Effects of hypoxia on the circadian patterns in men. High altitude medicine biology 2003;4(3):305-318.

[8] Hermida RC, Ayala DE, Portaluppi F. Circadian variation of blood pressure: the basis for the chronotherapy of hypertension. Advanced drug delivery reviews 2007; 59(9-10):904-922.

[9] Taylor KS, Heneghan CJ, Stevens RJ, Adams EC, Nunan D, Ward A. Heterogeneity of prognostic studies of 24-hour blood pressure variability: systematic review and meta-analysis. PLoS One 2015;10(5):e0126375.

[10] Johnson AE, Pollard TJ, Shen L, Li-wei HL, Feng M, Ghassemi M, Moody B, Szolovits P, Celi LA, Mark RG. MIMIC-III, a freely accessible critical care database. Scientific data 2016;3:160035.

[11] Saeed M, Villarroel M, Reisner AT, Clifford G, Lehman LW, Moody G, Heldt T, Kyaw TH, Moody B, Mark RG. Multiparameter Intelligent Monitoring in Intensive Care II (MIMIC-II): a public-access intensive care unit database. Critical care medicine 2011;39(5):952.

[12] Pollard TJ, Johnson AE, Raffa JD, Celi LA, Mark RG, Badawi O. The eICUfm collaborative research database, a freely available multi-center database for critical care research. Scientific data 2018;5.

[13] Johnson AE, Kramer AA, Clifford GD. A new severity of illness scale using a subset of acute physiology and chronic health evaluation data elements shows comparable predictive accuracy. Critical care medicine 2013; 41(7):1711-1718.

Address for correspondence:

Shaun Davidson

ORCRB, Roosevelt Drive, Oxford OX3 7DQ

shaun.davidson@eng.ox.ac.uk 